\title{
Grouping and Biological Toxicity Analyzing of Polybrominated Diphenyl Ethers based on Space Conformation and Molecular Symmetry
}

\author{
Long Jiang ${ }^{1, a}$ \\ ${ }^{1}$ North China Electric Power Research Institute Co Ltd. Beijing, 100045, China \\ akaveykikiy@163.com
}

\begin{abstract}
Keywords: polybrominated diphenyl ethers, biological toxicity, molecular symmetry, receptor binding affinities, quantitative structure-activity relationship.
\end{abstract}

\begin{abstract}
In this paper, the quantitative structure-activity relationship (QSAR) model of biological toxicity (-lgRBA) for 209 kinds of polybrominated diphenyl ethers (PBDEs) was established from view of substituents descriptors and quantum chemical parameters firstly. Then, they were divided into different groups based on their space conformation (regulated by substituent characters) and molecular symmetry firstly to analyze the relationship between biological toxicity and molecular symmetry of PBDEs. The results have shown that: the established QSAR model of biological toxicity possess good fitness, robustness and predictability for PBDEs based on selected independent variables; 209 congeners can be divided into 7 groups, and the specific biological toxicity sequence is: $\mathrm{G} 1$ (Group1) $<\mathrm{G} 3<\mathrm{G} 6<\mathrm{G} 5<\mathrm{G} 7<\mathrm{G} 2<\mathrm{G} 4$, because the steric hindrance of vertical configuration is not conducive to the binding with aryl hydrocarbon receptor (low biological toxicity).
\end{abstract}

\section{Introduction}

Polybrominated diphenyl ethers (PBDEs), an important class of flame retardant additives, have been used in household and industrial products widely [1-2]. PBDEs are consisted of 209 congeners depending on different number of $\mathrm{Br}$ atoms and different substituent positions among them. Because of the non-covalently attachment to polymer, PBDEs can escape from the products easily into environment [3]. Meanwhile, the characters of persistent organic pollutants (POPs) (persistence, bioaccumulation, long distance transportation via various approaches) have make PBDEs are now ubiquitous in urban and rural environment, producing significant negative effect on environment and health of organisms. Recent toxicity data suggested that PBDEs might have endocrine disrupting effects similar to halogenated organic compounds such as polychlorinated biphenyl (PCB) and dioxins [4-5].

The existing researches showed that the biological toxicity of majority halogenated hydrocarbons are regulated by the aryl hydrocarbon receptor (AhR) [6], which always could cause the dissociation of molecular chaperones, when after the AhR transferred to nucleus and changes the gene transcription, producing harm on organisms. The binding affinity of halogenated aromatic compounds to AhR (Receptor Binding Affinities, RBA) had been widely evaluating the degree of biological toxicity due to the positive correlation between them [7]. Compared to PCBs and dioxins, the studies reported on RBA of PBDEs were relatively few and the binding mechanism was vague. Different with PCBs, two benzenes rings of PBDEs is connected through ether bond, resulting worse space rotation and symmetry relative to PCBs, so the binding mechanism of PCBs to AhR could not be directly applied to PBDEs and it was necessary to analysis the biological toxicity varying rules among 209 PBDEs.

\section{Experimental and Methods}

\subsection{Experimental RBAs of PBDEs}

The experimental RBA values of 18 PBDEs were cited from literature [8]. Chen had tested the relative AhR binding affinities of each congener in rat hepatocytes, which were calculated relative to the $\mathrm{EC}_{50}$ values of the reference compound TCDD. For analysis conveniently, the RBA was 
converted as the negative of logarithm range, namely -lgRBA, which were listed in Table 1.

Table 1 Experimental -lgRBA of 18 PBDE congeners

\begin{tabular}{|c|c|c|c|}
\hline Congener & $-\lg$ RBA & Congener & $-\operatorname{lgRBA}$ \\
\hline BDE-3 & 3.89 & BDE-77 & 2.66 \\
\hline BDE-15 & 3.42 & BDE-85 & 1.72 \\
\hline BDE-17 & 3.64 & BDE-99 & 3.85 \\
\hline BDE-28 & 2.92 & BDE-100 & 4.11 \\
\hline BDE-47 & 3.25 & BDE-119 & 2.96 \\
\hline BDE-49 & 4.17 & BDE-126 & 2.57 \\
\hline BDE-66 & 2.70 & BDE-153 & 4.60 \\
\hline BDE-71 & 3.87 & BDE-154 & 4.64 \\
\hline BDE-75 & 3.40 & BDE-183 & 4.60 \\
\hline
\end{tabular}

\subsection{QSAR Modeling}

18 PBDEs were divided into model set (13 congeners) and testing set (5 congeners) by the fixed interval sampling method, and then the quantitative structure-activity relationship (QSAR) model of -lgRBA for PBDEs was established via partial least square (PLS). For the QSAR modeling, the substituent descriptors and quantum chemical parameters were selected as independent variables. The substituent descriptors contains: the total number of substituents $\left(N_{\mathrm{T}}\right)$, ortho-substituents number $\left(N_{2,6}, N_{2}, N_{6}\right)$, meta-substituents numbers $\left(N_{3,5}, N_{3}, N_{5}\right)$, para-substituents number $\left(N_{4}\right)$, the pair number of ortho-substituents $\left(N_{\mathrm{o}}\right)$, the pair number of meta-substituents $\left(N_{\mathrm{m}}\right)$, the pair number of para-substituents $\left(N_{\mathrm{P}}\right)$, the difference value of substituents between two rings $\left(N_{\mathrm{D}}\right)$, the sum of ortho-substituents and para-substituents $\left(N_{2,4,6}\right)$. The specific atom mark numbers of PBDEs were presented in Fig. 1.

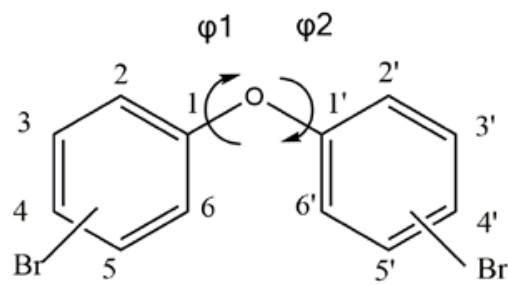

Fig. 1 Two torsion angles of PBDEs

The quantum chemical parameters were calculated via Gaussian 09W at the level of B3LYP/6-31G(d) based on the density functional theory. The specific parameters including: the dipole moment $(\mu)$, Quadrupole moment $\left(Q_{\mathrm{xx}}, Q_{\mathrm{yy}}, Q_{\mathrm{zz}}, Q_{\mathrm{xy}}, Q_{\mathrm{yz}}\right.$ and $\left.Q_{\mathrm{xz}}\right)$, frontier molecular orbitals ( $E_{\text {HOMO }}, E_{\text {LUMO }}, E_{\text {LUMO }}-E_{\text {HOMO }}$ ), Total energy $(T E)$, most negative atomic partial Mulliken charge $\left(q^{-}\right)$, most positive partial Mulliken charge $\left(q^{+}\right)$, the mean polarizability $(\alpha$,), the anisotropy polarisability $(\Delta \alpha)$ and approxpolarizability $\left(\alpha_{\mathrm{xx}}, \alpha_{\mathrm{yy}}, \alpha_{\mathrm{zz}}, \alpha_{\mathrm{xy}}, \alpha_{\mathrm{xz}}\right.$ and $\left.\alpha_{\mathrm{yz}}\right)$.

\subsection{Grouping of 209 PBDEs}

According to the degrees of two torsion angles of PBDEs, Schaefer et al. put forward four possible configurations: coplanar structure $\left(\varphi 1=\varphi 2=0^{\circ}\right)$, butterfly structure $\left(\varphi 1=\varphi 2=90^{\circ}\right)$, skew structure $\left(\varphi 1=0^{\circ}, \varphi 2=90^{\circ}\right)$ and distorted structure $\left(\varphi 1, \varphi 2>0^{\circ}\right)$ [9]. The degrees of two torsion angles are controlled by the number and position of substituents ( $\mathrm{Br}$ atoms), then regulate the space configuration and symmetry: when two substituents are located in ortho- 2 and ortho- 6 in same benzene ring at the same time, the degrees of two torsion angle for relative congeners are $90^{\circ}$ or $-90^{\circ}$, that is means two benzenes rings are orthogonal [10].

The molecular symmetry of PBDEs is related to three factors: the verticality between two benzenes, the substituent consistency between two benzenes and the symmetry of substituents in each benzene ring relative to ether bond. Then 209 congeners are divided into 7 groups by the screening sequence (Fig. 2). The grouping standards in Fig. 2 mean: S, the two rings have the same substituent pattern; D, the two rings have different substituent patterns; BS, the substituent patterns of both rings are symmetrical when treated as individual phenyl rings; SS, the substituent pattern of each ring is symmetrical when treated as an individual phenyl ring; NS, the substituent patterns of both rings are asymmetric when treated as individual phenyl rings; $\mathrm{P}$, the two phenyl rings are perpendicular to each other; NP, the two phenyl rings were not perpendicular to each other. 


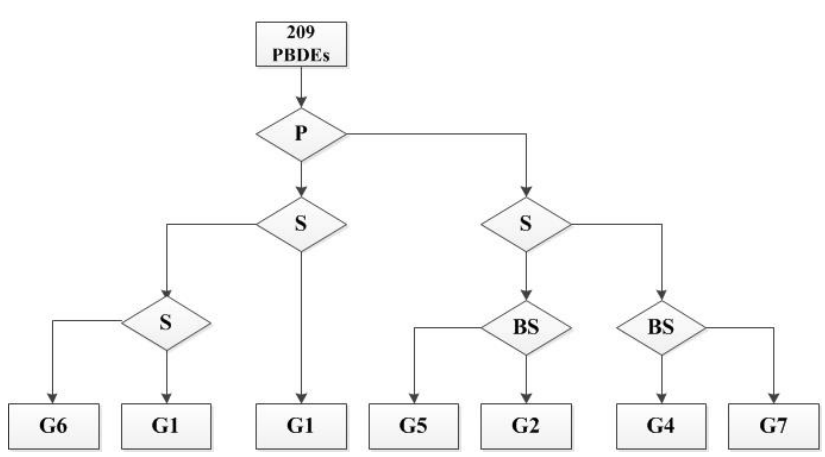

Fig. 2 Grouping flowcharts of 209 PBDEs based on space conformation and molecular symmetry

\section{Results and Discussion}

\subsection{The Established QSAR Model}

Using the modeling approach introduced above, the independent variables were screened constantly by variable importance projection (VIP) of each variable to obtain the optimal QSAR model, presented as follow:

$$
-\lg \mathrm{RBA}=17.102-83.491 q H^{+}+0.724 N_{2,6}+0.508 N_{5}+0.307 N_{\mathrm{P}}
$$

For the performance validation of the established model: $R_{\mathrm{x}}^{2}=0.901, R_{\mathrm{Y}}^{2}=0.944, R^{2}=0.928(>0.85)$, $q^{2}=0.783(>0.50), P R E S S=1.421, F=42.87$ (sig. $\left.=0.000\right), R_{\text {pre }}^{2}=0.828(>0.80)$, presenting the QSAR model possesses well fitness, robustness and predictability.

\subsection{Grouping of 209 PBDEs}

Based on the grouping flowcharts in Fig. 2, 209 congeners are divided into 7 groups (listed in Table 2) which are same to the symmetry group of PCBs, presenting the symmetry property-preserving of PBDEs from PCBs although 209 PBDEs all belong to symmetry level of Cs.

Table 2 Grouping of PBDEs based on space conformation and molecular symmetry

\begin{tabular}{|c|c|c|c|}
\hline Group & $\begin{array}{l}\text { Group } \\
\text { Standard }\end{array}$ & Amount & PBDEs \\
\hline 1 & $\mathrm{~S}+\mathrm{BS}+\mathrm{P}$ & 4 & 54、155、202、209 \\
\hline 2 & $\mathrm{~S}+\mathrm{BS}+\mathrm{NP}$ & 3 & 15、80、169 \\
\hline 3 & $\mathrm{D}+\mathrm{BS}+\mathrm{P}$ & 22 & $\begin{array}{l}10 、 30 、 32 、 65 、 73 、 75 、 104 、 116 、 117 、 121 、 125 、 152 、 165 、 \\
166 、 168 、 186 、 188 、 192 、 193 、 204 、 205 、 208\end{array}$ \\
\hline 4 & $\mathrm{D}+\mathrm{BS}+\mathrm{NP}$ & 6 & 3、14、38、39、81、127 \\
\hline 5 & $\mathrm{~S}+\mathrm{NS}+\mathrm{NP}$ & 12 & 4、11、40、47、52、77、128、133、136、153、194、197 \\
\hline 6 & $\mathrm{D}+\mathrm{NS}+\mathrm{P}$ & 56 & $\begin{array}{l}19 、 24 、 27 、 46 、 50 、 51 、 53 、 62 、 64 、 69 、 71 、 89 、 93 、 94 、 \\
96 、 98 、 100 、 102 、 103 、 112 、 113 、 115 、 119 、 134 、 140 、 142 、 \\
143,145 、 147 、 148 、 150 、 151 、 154 、 160 、 161 、 163 、 164 、 173 、 \\
177 、 178 、 179 、 181 、 182 、 184 、 185 、 187 、 190 、 191 、 195 、 198 、 \\
199 、 200 、 201 、 203 、 206 、 207 \\
\end{array}$ \\
\hline 7 & $\begin{array}{l}\text { D+SS/NS } \\
+\mathrm{NP}\end{array}$ & 106 & $\begin{array}{l}1 、 2 、 5 、 7 、 9 、 12 、 21 、 23 、 29 、 61,62 、 8 、 13 、 16 、 17 、 18 、 \\
20 、 22 、 25 、 26 、 28 、 3133 、 34 、 35 、 36 、 37 、 41 、 42 、 43 、 44 、 \\
45 、 48 、 49 、 55 、 56 、 57 、 58 、 59 、 60 、 63 、 66 、 67 、 68 、 70 、 \\
72 、 74 、 76 、 78 、 79 、 82 、 83 、 84 、 85 、 86 、 87 、 88 、 90 、 91 、 \\
92 、 95 、 97 、 99 、 101 、 105 、 106 、 107 、 108 、 109 、 110 、 111 、 \\
114 、 118 、 120 、 122 、 123 、 124 、 126 、 129 、 130 、 131 、 132 、 135 、 \\
137 、 138,139 、 141 、 144 、 146 、 149 、 156 、 157 、 158 、 159 、 162 、 \\
167 、 170 、 171 、 172 、 174 、 175 、 176 、 180 、 183 、 189 、 196\end{array}$ \\
\hline
\end{tabular}

\subsection{Biological Toxicity Analyzing Based on Space Conformation and Molecular Symmetry}

Through the data analysis and the predicted -lgRBA of 209 PBDEs, 45 kinds PBDEs among the smallest biological toxicity of 50 congeners $(-\lg \mathrm{RBA}>5)$ have vertical conformation; meanwhile, the largest biological toxicity of 20 congeners all have non-vertical conformation. This is mainly due to steric hindrance for vertical configuration, which is not conducive to the binding with aryl 
hydrocarbon receptor (low biological toxicity). According the symmetry grouping in Table 2, the biological toxicity comparison among different groups was shown in Fig. 3. The -lgRBA of Groups 1, 3, 6 are larger than other due to their vertical conformation, and the specific biological toxicity sequence for 7 groups is: $\mathrm{G} 1$ (Group1) $<\mathrm{G} 3<\mathrm{G} 6<\mathrm{G} 5<\mathrm{G} 7<\mathrm{G} 2<\mathrm{G} 4$. Furthermore, combined with the symmetrical structure and substituent characters we can see: when the substituent character for two benzene rings is different, the corresponding PBDEs (G1, G2, G3, G4) always possess the largest or smallest biological toxicity, and the extremal direction was decided by the verticality; for vertical conformation (G1, G3), congeners with smaller biological toxicity; for non-vertical conformation (G2, G4), congeners with larger biological toxicity. From the view of molecular symmetry, there is no obvious relationship between relative symmetric level (referenced from PCBs) and biological toxicity from Fig. 3.

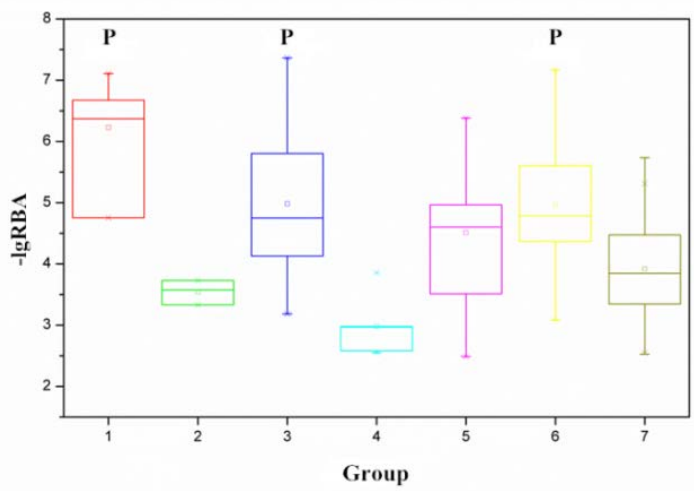

Fig.3 Comparison among different groups

\section{Summary}

(1) Using the PLS method, the established QSAR model of biological toxicity (-lgRBA) for 209 PBDEs has shown well good fitness, robustness and predictability, with $R^{2}$ and $q^{2}$ are more than 0.85 and 0.50 respectively.

(2) 209 congeners can be divided into 7 groups based on their s space conformation and molecular symmetry; groups with vertical configuration (G1, G3, G6) always have low biological toxicity because their steric hindrance between two benzenes rings is not conducive to the binding with aryl hydrocarbon receptor; there is no obvious relationship between relative symmetric level and biological toxicity.

\section{References}

[1] P.R. Erickson, M. Grandbois, W.A. Arnold and K. McNeill, Photochemical formation of brominated dioxins and other products of concern from hydroxylated polybrominated diphenyl ethers (OH-PBDEs). Environ. Sci. Technol. 46 (2012) 8174-8180.

[2] I. Labunska, S. Harrad, D. Santillo, P. Johnston and K. Brigden, Levels and distribution of polybrominated diphenyl ethers in soil, sediment and dust samples collected from various electronic waste recycling sites within Guiyu town, southern China. Environ. Science: Processes \& Impact. 15 (2013) 503-511.

[3] S. Na, M. Kim, O. Paek and Y. Kim, Dietary assessment of human exposure to PBDEs in South Korea. Chemosphere 90 (2013) 1736-1741

[4] A.K. Peters, J.T. Sanderson, A. Bergman and M. van der Berg, Antagonism of TCDD-induced ethoxyresorufin-O-deethylation activity by polybrominated diphenyl ethers (PBDEs) in primary cynomolgus monkey (Macaca fascicularis) hepatocytes. Toxicol. Lett. 164 (2006) 123-132.

[5] M. Wahl, B. Lahni, R. Guenther, B. Kuch, L. Yang, U. Straehle, S. Strack and C. Weiss, A technical mixture of 2,2',4,4'-tetrabromo diphenyl ether (BDE47) and brominated furans triggers 
aryl hydrocarbon receptor (AhR) mediated gene expression and toxicity. Chemosphere 73 (2008) 209-215.

[6] Y.Z. Gu, J.B. Hogenesch and C.A. Bradfield, The PAS superfamily: sensors of environmental and developmental signals. Ann. Rev. Pharmacol. Toxical. 40 (2000) 519-561.

[7] S. Kovarich, E. Papa and P. Gramatica, QSAR classification models for the prediction of endocrine disrupting activity of brominated flame retardants. J. Hazard. Mater. 190 (2011) 106-112.

[8] G.S. Chen, A.D. Konstantinov, B.G. Chittim, E.M. Joyce, N.C. Bols and N.J. Bunce, Synthesis of polybrominated diphenyl ethers and their capacity to induce CYP1A by the Ah receptor mediated pathway. Environ. Sci. Technol. 35 (2001) 3749-3756.

[9] T. Schaefer, G.H. Penner and C. Takeuchi, Remarks on the internal motion in diphenyl ether. Fluorophenyl ethers. Can. J. Chem. 66 (1988) 1647-1650

[10] G. Luthe, D.C. Swensen, Influence of fluoro-substitution on the planarity of 4-chlorobiphenyl (PCB 3). Acta Crystallogr. 63 (2007) 319-327 